\title{
Characteristics of Lactobacillus plantarum QZW5 and its effects on wheat silage under multigelation
}

\author{
Miao Zhang ${ }^{1}$, Guofang Wu², Lei Wang², Bei Zhang ${ }^{3}$, Jun Chen ${ }^{1}$, Yuan Liu' ${ }^{1}$, Huili Pang ${ }^{1}$ and Zhongfang Tan ${ }^{\text {** }}$
}

\begin{abstract}
Background: Silages, as a nutritious and long-term preserved fodder, is achieved through fermentation. However, the constant low temperatures and freeze-thaw event are key factors that affect the quality of silages due to low levels of lactic acid production and undesirable microbial community revitalization. In this study, we attempt to investigate the effects of Lactobacillus plantarum QZW5 on the fermentation characteristics of wheat silage under ensiling conditions at the order of the following temperatures: constant low temperatures $\left(5^{\circ} \mathrm{C}\right.$ for 30 days), multigelation $\left(10^{\circ} \mathrm{C}\right.$ at day and $-10^{\circ} \mathrm{C}$ at night for 30 days) and room temperature (aerobic/anaerobic, 10 days).

Results: Under multigelation condition, Lactobacillus plantarum QZW5 inoculation significantly increased the contents of lactic acid and acetic $(P<0.05)$ in the silage. The $\mathrm{pH}$, bacterial richness index (Chao) and diversity index (Shannon) of the silage were significantly decreased by Lactobacillus plantarum QZW5 $(P<0.05)$. In Lactobacillus plantarum QZW5 inoculated silage, the dominant genera were L. plantarum subsp. plantarum. However, those in the control silage were Leuconostoc mesenteroides and Leuconostoc fallax. LAB (lactic acid bacteria) inoculation increased the abundance of desirable Lactobacillus spp. and inhibited the growth of undesirable Escherichia coli, Saccharomyces cerevisiae, and Filamentous fungi, among others in the silage.
\end{abstract}

Conclusions: Therefore, inoculation of L. plantarum QZW5 during ensiling could stimulate favorable fermentation and reconstruct bacterial community for better silage preservation.

Keywords: Lactobacillus plantarum QZW5, Low temperature, Multigelation, Wheat, Silage

\section{Introduction}

Ensiling, as a popular method for plant material storage under acidic conditions, can ferment plant sugars to lactic acids using lactic acid bacteria (LAB) to achieve long-term nutrients preservation of unfermented feed. Moreover, the silage can keep higher nutritional values than that of green hay. However, the poor quality of silages can be induced by recurrent freezing and thawing with the change of seasons (winter/spring). The lack of adequate levels of lactic acid production is one of the

\footnotetext{
*Correspondence: tzhongfang@zzu.edu.cn

${ }^{1}$ Henan Key Laboratory of Ion-Beam Bioengineering, College

of Agricultural Sciences, Zhengzhou University, Zhengzhou, China

Full list of author information is available at the end of the article
}

key points $[1,2]$. To overcome this problem, the addition of an acid-producing agent to silage could be a feasible strategy.

Previous studies have found that culture conditions such as temperature and $\mathrm{pH}$ could alter the cell membrane properties of microorganisms [3], such as flexibility, cell adhesion, metabolism, permeability, membrane structures, and also fatty acid composition, and could affect heat resistance of microorganisms [4]. Microorganisms thrive in specific habitats and vary with geographical environment [5]. The bacteria isolated from the unique ecosystem of the Qinghai-Tibet Plateau with strong stress tolerance are considered to have potential application in the agriculture, medicine, aquaculture, and food industry $[6,7]$. It has been reported that LAB, 
who has characteristics, including tolerance to stress conditions (e.g., acidic/alkaline conditions, high/low temperature, etc.) and capability of producing compounds against undesirable microorganisms, could be employed as microbial additives to prevent spoilage of silages [8]. LAB-related additives have been proved that they can significantly improve the fermentation quality of silages and inhibited its nutrition losing [9]. Therefore, find a suitable LAB-related additive combination for recurrent freezing and thawing conditions has crucial role in silages quality.

Lactobacillus plantarum QZW5 was isolated from the Qinghai-Tibet Plateau and has used as an LAB additive. In this study, we aimed to determine the effects of $\mathrm{Lac}$ tobacillus plantarum (renamed as Lactiplantibacillus plantarum) QZW5 on the fermentation profile and bacterial community of wheat silage. The ensiling conditions simulated were constant low temperatures, followed by repeated freezing and thawing at low temperatures according to the temperature variations of Hainan Tibetan Autonomous Prefecture (Additional file 1: Fig. S1). The aims of this study were to: (i) measure the levels of acid in silage by effective fermentation with Lactobacillus plantarum QZW5; (ii) determine the inhibitory effect of Lactobacillus plantarum QZW5 on spoilage microorganisms under multigelation, and (iii) provide an effective manner for establishing quality control of silage in winter. The findings of this study could help to improve the utilization of existing feed resources.

\section{Materials and methods \\ Additives selection}

The permission of collection LAB samples from the sampling location was issued by Zhengzhou University, Zhengzhou, China. The LAB isolates were preserved at $-80{ }^{\circ} \mathrm{C}$. The strain QZW5 was isolated from traditional dairy products of the Qinghai-Tibet Plateau at an altitude of 3100-4800 m, which has finished the morphological, physiological, and biochemical tests and has been selected as LAB additive (Table 1). The tolerance of strain QZW5 to $\mathrm{NaCl}$, bile, acid, alkali, and low and high-temperature stress was measured. The bacterial growth rates were assayed based on visual turbidity at $550 \mathrm{~nm}$ [10]. The antimicrobial activity of strain QZW5 was measured according to the size of inhibition zone using bidirectional diffusion method. As shown in Table 1, strain QZW5 was isolated from dairy products in Gonghe County, Qinghai Province, China, which was identified as L. plantarum using $16 \mathrm{~S}$ rRNA-based phylogeny and typical morphophysiological characteristics.

Table 1 Morphological, physiological, and biochemical properties of strain QZW5

\begin{tabular}{|c|c|}
\hline Character & QZW5 \\
\hline Species & Lactobacillus plantarum \\
\hline Collecting location & Gonghe county, Qinghai, China \\
\hline Sample sources & Dairy products \\
\hline Shape & Rod \\
\hline Gram stain & + \\
\hline Catalase & - \\
\hline Gas from glucose & - \\
\hline Fermentation type & Homo \\
\hline \multicolumn{2}{|l|}{ Growth at temp $\left({ }^{\circ} \mathrm{C}\right)$ : } \\
\hline $5,10,45,50$ & + \\
\hline \multicolumn{2}{|l|}{ Growth in $\mathrm{NaCl}$ : } \\
\hline $3.0 \%, 6.5 \%$ & + \\
\hline \multicolumn{2}{|l|}{ Growth in tile } \\
\hline $0.10 \%, 0.30 \%$ & w \\
\hline \multicolumn{2}{|l|}{ Growth at pH: } \\
\hline 2 & w \\
\hline $3-10$ & + \\
\hline \multicolumn{2}{|l|}{ Pathogen inhibition } \\
\hline Micrococcus luteus ATCC 4698, Bacillus subtilis ATCC 6633 & +++ \\
\hline Staphylococcus aureus ATCC 29213, Pseudomonas aeruginosa ATCC 27853, Salmonella enterica ATCC14028 & ++ \\
\hline Escherichia coli ATCC13076 & + \\
\hline
\end{tabular}

+ Good growth, w weak growth. The inhibition zone spans the external diameter of Oxford cup ( $7.68 \mathrm{~mm})$. Inhibition zone: $<8.00 \mathrm{~mm},-; 8.00-12.00 \mathrm{~mm},+$; $12.00-16.00 \mathrm{~mm},++;>16.00 \mathrm{~mm},+++$ 
Strain QZW5 presented excellent high and low-temperature tolerance and could grow well at $5,10,45$, and $50{ }^{\circ} \mathrm{C}$. Furthermore, strain QZW5 also showed excellent tolerance to $\mathrm{NaCl}$ and bile, respectively, and could grow well in $3.0-6.5 \% \mathrm{NaCl}$ and $0.1-0.3 \%$ bile. Moreover, strain QZW5 could thrive under extremely acidic conditions of $\mathrm{pH} 2.0$ and extremely alkaline conditions of $\mathrm{pH}$ 10.0. In addition, strain QZW5 exhibited broadspectrum antibacterial activity, exerting inhibitory effect on both Gram-negative pathogens, including $E$. coli, Pseudomonas aeruginosa, and Salmonella enterica, and Gram-positive pathogens, such as Micrococcus luteus, Bacillus subtilis, and Staphylococcus aureus.

\section{Ensiling process}

The hexaploid wheat plants, which were harvested at dough stage, were used as unfermented materials (Henan Key Laboratory of Ion-Beam Bioengineering, Zhengzhou University). These materials were shredded to pieces $(2-3 \mathrm{~cm}$ in long) after wilted about $10 \mathrm{~h}$. QZW5 was revitalized with de Man, Rogosa, and Sharpe (MRS) agar medium and then inoculated into liquid MRS broth for $24 \mathrm{~h} .24 \mathrm{~kg}$ of the shredded wheat was inoculated with $1600 \mathrm{~mL}$ QZW5 and thoroughly mixed (QZW5 group). The final inoculation dose of the revitalized viable QZW5 in the wheat plants was $6.82 \mathrm{log} \mathrm{CFU} / \mathrm{g}$. In the control group, $1600 \mathrm{~mL}$ distilled water was added into $24 \mathrm{~kg}$ of wheat. For each of these two treatments we collected from the pile and ensiled it. A total of $800 \mathrm{~g}$ of the material were collected into a plastic film bag (in triplicate) (Dragon N-6; Asahi Kasei Co., Tokyo, Japan) and sealed using a vacuum sealer (SQ-203S Vacuum Sealer; Asahi Kasei Co).

According to the temperature variations (Additional file 1: Fig. S1) in Hainan Tibetan Autonomous Prefecture (Qinghai, China), the annual average temperature was $5.7^{\circ} \mathrm{C}$. The average minimum temperature at night and maximum temperature at day in winter were $-8{ }^{\circ} \mathrm{C}$ and $8{ }^{\circ} \mathrm{C}$, respectively. The maximum air temperature in summer was about $30{ }^{\circ} \mathrm{C}$. In our laboratory study, the packed silage bags were fermented for 30 days at constant low temperature $\left(5^{\circ} \mathrm{C}\right)$, and then preserved in a refrigerator at $10{ }^{\circ} \mathrm{C}$ during the day and in a freezer at $-10^{\circ} \mathrm{C}$ at night alternately for 30 days. Subsequently, the silages were placed at room temperature of $25{ }^{\circ} \mathrm{C}$ under aerobic (open silage bags) and anaerobic (airtight silage bags) condition for 10 days, respectively, to test temperature stability and aerobic stability. The entire ensiling process lasted 70 days. The microbial detection, microbial genome sequencing and chemical analyses were performed every 10 days.

\section{Viable microorganisms}

The viable microbial community was cultured and enumerated on different culture media by dilution separation method [11-13]. LAB, aerobic bacteria, yeasts and molds, and Escherichia coli were cultured and enumerated in MRS medium; nutrient agar medium, potato dextrose agar medium, and eosin methylene blue medium, respectively. All the colonies were counted and the logarithmic number of viable colony-forming (CFU) units in fresh matter was calculated.

\section{Nutritional components of silages}

A total of $10 \mathrm{~g}$ of silage was mixed with $90 \mathrm{~mL}$ of deionized water. About $50 \mathrm{~mL}$ mixture were filtered through qualitative filter paper and then used for ammonia nitrogen $\left(\mathrm{NH}_{3}-\mathrm{N}\right)$ and organic acid analyses. The remaining mixture was used for $\mathrm{pH}$ detection using an electronic pH meter (Mettler Toledo MP230; Greifensee, Switzerland). The $\mathrm{NH}_{3}-\mathrm{N}$ content was measured using indoxyl blue spectrophotometric method, and the organic acid content was determined with high performance liquid chromatography [2]. The dry matter (DM), crude ash and crude protein were assayed by atmospheric pressure drying method (AOAC 934.01), high-temperature ashing method (AOAC 942.05), and Kjeldahl method (AOAC 979.09), respectively.

The remaining silage was further dried in a thermostatic blast furnace (Shanghai Shuli Instrument Co., Ltd., Shanghai, China) for 2 days at $65{ }^{\circ} \mathrm{C}$ and the percentage DM (\% DM) and moisture content (\% FM) were calculated.

The dried silage was smashed using a high-speed pulverizer (FW-100, Taisite Instrument Co. Ltd., Jinghai, Tianjin, China). The crude protein content in the silage powder was detected using automatic Kjeldahl apparatus (K1100, Jinan Hanon Instrument Co. Ltd., Jinan, China). The standard concentration of the titrated $\mathrm{H}_{2} \mathrm{SO}_{4}$ solution was determined by color indicator titration method [14]. The crude ash content was calculated after incineration for $2 \mathrm{~h}$ at $550{ }^{\circ} \mathrm{C}$ in a muffle furnace (REX-C900, RKC Instrument Inc., Osaka, Japan) and cauterized on an electric furnace until smokeless. The detergent fiber was determined by Automatic Fiber Determination System (CXC-06, Zhejiang Top Instrument Ltd., Hangzhou, China).

\section{Microbial abundance composition}

$10 \mathrm{~g}$ refrigerated silage was mixed with $40 \mathrm{~mL}$ sterile deionized water in an electronic oscillator at $120 \mathrm{rpm}$ for $2 \mathrm{~h}$ and then filtered using double gauze masks. The filtrate was centrifuged for $15 \mathrm{~min}$ at $12,000 \times g$ and $4{ }^{\circ} \mathrm{C}$, 
and the solids were collected and preserved in dry ice. Metagenomics Sequencing was performed by Majorbio Bio-Pharm Technology Co. Ltd. (Shanghai, China) based on the following steps: (i) DNA extraction and PCR amplification; (ii) Illumina MiSeq sequencing; (iii) processing of the sequencing data. Operational taxonomic units (OTUs) with a 97\% similarity cutoff were clustered using UPARSE version 7.1 (http://drive5.com/uparse/). The similarity of sequences which was of below $97 \%$ were removed [15]. The taxonomy of each OTU representative sequence was analyzed by RDP Classifier (http://rdp. cme.msu.edu/) against the $16 \mathrm{~S}$ rRNA database (e.g., Silva SSU128) using 0.7 as confidence threshold $[16,17]$.

\section{Statistical analyses}

The inoculation effect at the whole fermentation time was conducted by two-way analysis of variance. The inoculation effect at each fermentation time was examined by one-way analysis of variance, followed by multiple comparison by Tukey's test. The statistical significances of the antibacterial activity and $\mathrm{NH}_{3}-\mathrm{N}$-producing ability of pathogens were evaluated using ANOVA with Tukey's HSD test (IBM SPSS Statistics 19 software; https://www. ibm.com/analytics/cn). The $\mathrm{pH}$ variations and microbial communities were plotted using Origin software Pro 8.5.1 (http://www.originlab.com/index.aspx?go=Produ cts/Origin). The bacterial and fungal sequencing data were analyzed using the free online Majorbio Cloud Platform (www.majorbio.com).

\section{Results and discussion}

\section{Characteristics of strain QZW5}

It has been reported that the organic acid produced by $L A B$ exhibits resistant effects on certain pathogens. As shown in Fig. 1a, after $48 \mathrm{~h}$ of cultivation, the $\mathrm{pH}$ value of the strain QZW5 culture medium decreased to 3.76, and the optical density (OD) values increased to 1.25 . It showed a significant negative correlation between OD and $\mathrm{pH}$ during cultivation. The concentrations of lactic acid and acetic acid produced by strain QZW5 after $48 \mathrm{~h}$ cultivation were 45.07 and $9.13 \mathrm{mg} / \mathrm{mL}$, respectively (Fig. 1b). Propionic and butyric acids were not detected in strain QZW5 culture medium.

\section{Properties of the unfermented materials}

The chemical composition and microbial community of the unfermented materials wilted are shown in Table 2 . The $\mathrm{pH}$ of the wilted unfermented materials was 6.57 .

Table 2 Chemical composition and microbial community of wilted unfermented materials prior to ensiling

\begin{tabular}{ll}
\hline & Wheat \\
\hline pH & $6.57 \pm 0.17$ \\
Moisture (\% FM) & $63.24 \pm 0.28$ \\
Dry matter (\% FM) & $36.76 \pm 0.28$ \\
Crude protein (\% DM) & $8.65 \pm 0.12$ \\
Crude fat (\% DM) & $12.51 \pm 0.75$ \\
NDF (\% DM) & $60.18 \pm 2.21$ \\
ADF (\% DM) & $34.12 \pm 1.55$ \\
Crude ash (\% DM) & $7.33 \pm 0.64$ \\
Organic acid (mg/g DM) & \\
Lactic acid, acetic acid, butyric acid & nd \\
Viable microbiological counts (log CFU/g FM) & \\
Lactic acid bacteria & $1.89 \pm 0.47$ \\
E. coli & $2.15 \pm 0.19$ \\
F. fungi & $2.00 \pm 0.65$ \\
Aerobic bacteria & $3.57 \pm 0.52$ \\
S. marcescens, S. cerevisiae, B. subtilis & nd \\
\hline
\end{tabular}

nd not detected, $D M$ dry matter of wilted unfermented materials before inoculating
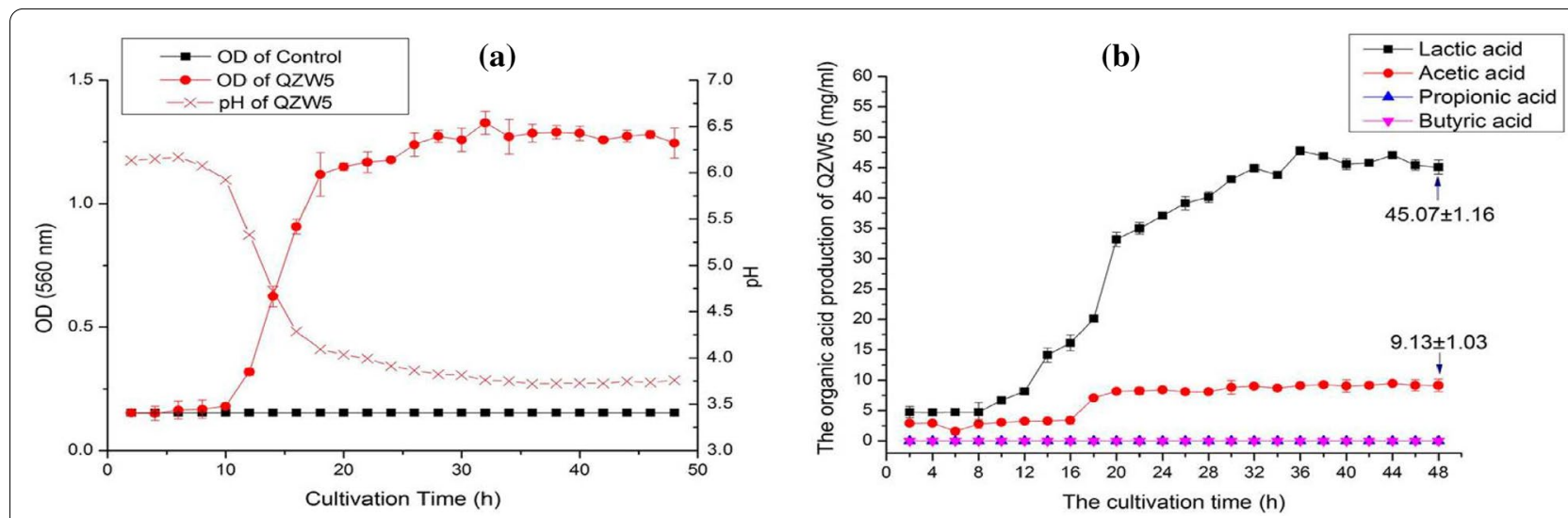

Fig. 1 The $\mathrm{pH}$ (a) and organic acid (b) variations of QZW5 cultured individually 
Moisture content was $63.24 \%$. FM, crude protein and ether extract contents were $8.65 \% \pm 0.12 \%$ DM (dry matter of wilted unfermented materials before inoculating, similarly hereinafter) and $12.51 \% \pm 0.75 \%$ dry matter, respectively. Neutral detergent fiber (NDF) and acid detergent fiber (ADF) contents were $60.18 \% \pm 2.21 \%$ DM and $34.12 \% \pm 1.55 \% \mathrm{DM}$, respectively. Previous studies have shown that the moisture, crude protein, fat, $\mathrm{NDF}$, and ADF contents in silage do not significantly change overall [2]. With regard to organic acid production, no organic acid was detected in the present study. Epiphytic LAB counts in the unfermented materials were $1.89 \pm 0.47 \log$ CFU/g FM, and the results of highthroughput sequencing (Fig. 4) revealed that the predominant epiphytic LAB in the unfermented materials were L. plantarum subsp. plantarum and Leuconostoc mesenteroides. Besides, E. coli, Filamentous fungi (F. fungi), and aerobic bacteria were also detected in the unfermented materials, whereas S. marcescens (Serratia marcescens), S. cerevisiae (Saccharomyces cerevisiae), and B. subtilis (Bacillus subtilis) were not found.

\section{Chemical components and $\mathrm{pH}$ variations of silage}

As shown in Table 3, the $\mathrm{pH}$ of silage inoculated with QZW5 reduced to 4.26 on day 30 and continued at an approximate low value in the subsequent process of preservation. In contrast, the $\mathrm{pH}$ of silage of the control group without bacterial inoculation was undesirably above 6.17 during the entire experimental period at low temperature (from -10 to $10{ }^{\circ} \mathrm{C}$ ). It has been reported that fermentation parameters, such as $\mathrm{pH}$, volatile fatty acids content, and $\mathrm{NH}_{3}-\mathrm{N}$ levels, influence energy intake and subsequent milk yield [18], and that higher $\mathrm{pH}$ is often accompanied by higher $\mathrm{NH}_{3}-\mathrm{N}$ content, which also indicates poorer preservation quality and lower feed intake. Moist silage with a high $\mathrm{pH}$ of $>4.2$ is difficult to preserve for an extended period of time, and the activities of spoilage bacteria and butyric acid bacteria are generally more intense during the silage fermentation process at $\mathrm{pH}>4.4$ (except for low-moisture silage). In the present study, at variable low temperature from days 10 to 60 , the $\mathrm{pH}$ of silage of the control group without bacterial inoculation was unfavorable for storage, whereas that of silage of the QZW5 group was favorable for preservation comparatively.

During the ensiling process, LAB convert carbohydrates in wheat into organic acids. Organic acids produced by LAB are lactic acid, acetic acid and propionic acid. lactic acid improved the silage preservation by causing a rapid drop in $\mathrm{pH}[19,20]$. The aroma and flavor of the feed increase with increasing acetic acid content [21], acetic acid or propionic acid, thereby inhibiting the growth of spoilage yeasts and molds and improving the aerobic stability of silage [19, 22, 23]. The microbial population, inoculum, substrate complexity, nutrients availability, $\mathrm{pH}$, and temperature have all been noted to affect organic acids production [24]. In the present study, lactic acid was not detected in the control group during the variable low-temperature fermentation stage. In contrast, in the QZW5 group, the lactic acid content increased to $3.36 \pm 1.17$ and $4.85 \pm 1.18 \mathrm{mg} / \mathrm{mL}$ on days 30 and 40 , respectively, during the continuous low-temperature fermentation $\left(5^{\circ} \mathrm{C}\right)$ stage, but decreased to $3.30 \pm 0.64 \mathrm{mg} /$ $\mathrm{mL}$ following temperature variation, indicating that strain QZW5 was helpful for preservation.

The acetic acid contents in the QZW5 and control groups were not significantly different throughout the entire fermentation process, and propionic acid was not detected during continuous low-temperature fermentation and the subsequent variable temperatures stage. It has been reported that propionic acid is produced in silage when the temperature rises to $25^{\circ} \mathrm{C}$ under anaerobic conditions and that certain heterofermentative $\mathrm{LAB}$ produce high concentrations of acetic or propionic acid, which inhibit the growth of spoilage yeasts and molds and improve the shelf life of silage during feedout [27], but do not affect the relative abundances of predominant bacteria, such as Lactobacillus, Weissella, or Pediococcus [28]. In a previous study, propionic acid was added as an antifungal agent to enhance the shelf life of silages during feedout [29]. Propionic acid easily accumulates in the organic anaerobic digestion system [25], the content of propionic acid in the QZW5 group was higher than the control group in anaerobic conditions at $25^{\circ} \mathrm{C}$, although the increase was not significant $(P \geq 0.05)$.

At low temperature from days 10 to 60 , the DM content in the control group was significantly higher than that in the QZW5, the DM variations in present study is not consistent with the previous publications. Inoculation of psychrotrophic L. plantarum has been noted to reduce the moisture content of silage at low temperature, decreasing DM loss during preservation [30]. However, in the present study, strain QZW5 consumed more DM and produced more organic acid at low temperature. Furthermore, the $\mathrm{NH}_{3}-\mathrm{N}$ content in the QZW5 group was significantly lower than that in the control group during the ensiling process $(0.01<P<0.05)$.

\section{Viable microorganisms}

During the natural ensiling process, epiphytic LAB on the plant surface play a critical role in the quality of the final silage. As shown in Fig. 2, F. fungi and E. coli were detected in the control group after fermentation for 30 days, whereas neither of these microorganisms were found in the QZW5 group. On day 50, S. cerevisiae and E. coli were noted in the control group, but were not 


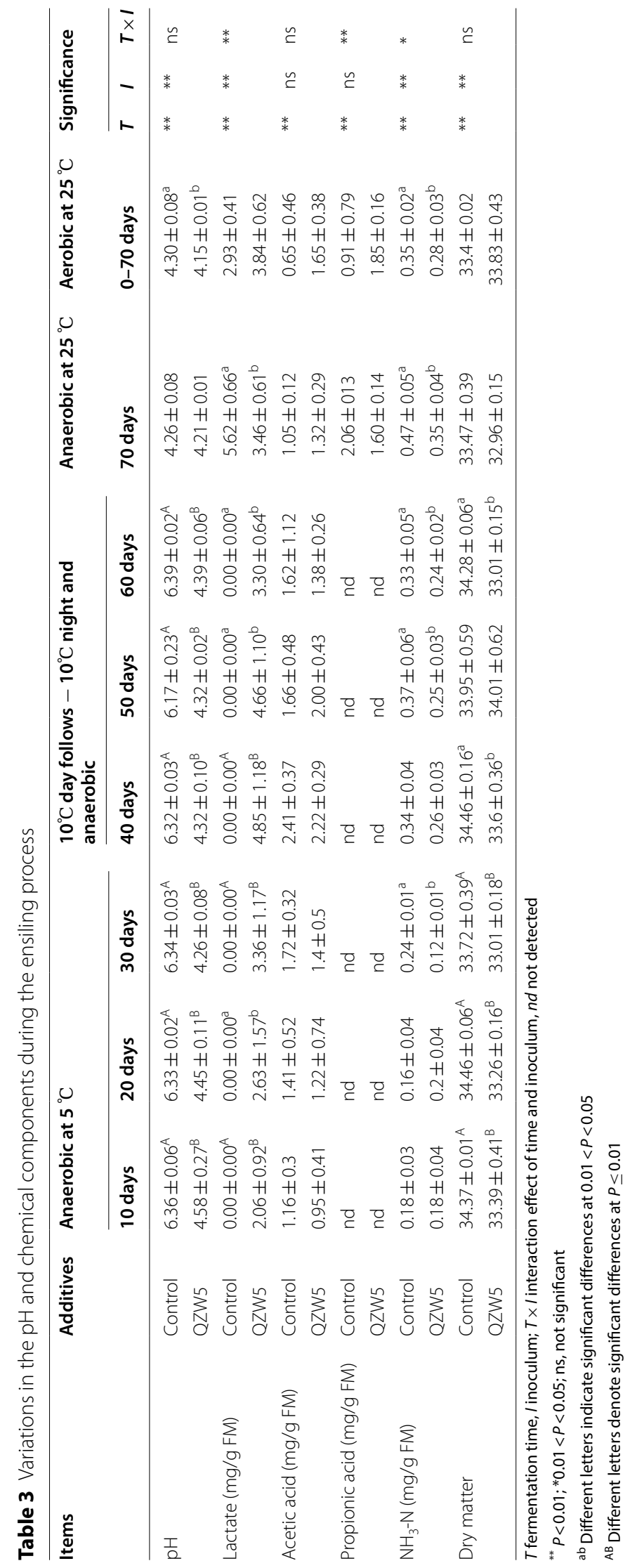



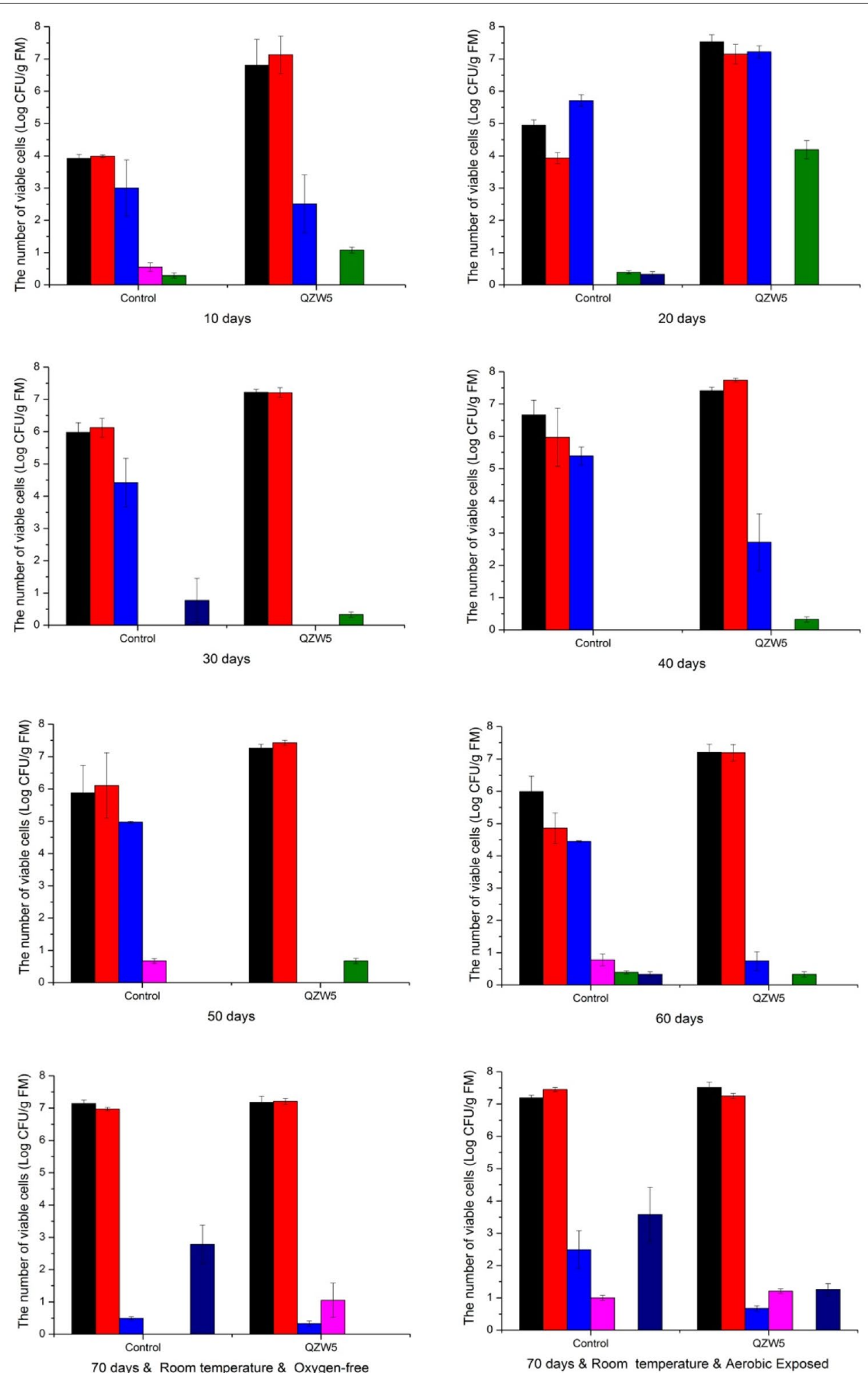
$\begin{array}{ll}\text { Acidophilus } & \text { A.bacteria } \\ \text { Saccharomycetes } & \text { B.subtilis } \\ \text { E.coli }\end{array}$

Fig. 2 The counts of viable cells 
detected in the QZW5 group. These results, along with the antibacterial activity findings (Table 1), demonstrated that strain QZW5 has broad-spectrum antibacterial activity and could effectively inhibit pathogens.

The proliferation of yeasts, molds, and other undesirable pathogens not only induces nutrients loss, but also the production of mycotoxins have potential negative effects on animal and human health [26]. In our study, under aerobic condition for 10 days, the quantities of $F$. fungi and E. coli were lower than those noted in the control group when temperature was increased to $25{ }^{\circ} \mathrm{C}$. Under anaerobic condition with the increase in temperature to $25^{\circ} \mathrm{C}$ for 10 days, counts of E. coli were lower than control group, $F$. fungi were detected in control group but not in QZW5. Thus, it can be concluded that strain QZW5 inhibited the number of viable F. fungi and E. coli in silage under aerobic/anaerobic conditions and atmospheric temperature.

\section{Microbial abundances}

The relative bacterial abundances in silage are shown in Fig. 3a. The dominant epiphytic bacteria in unfermented wheat were Pantoea agglomerans, Pseudomonas and Frigoribacterium. In the first 30 days of constant low-temperature fermentation, L. plantarum subsp. plantarum promoted the dominant

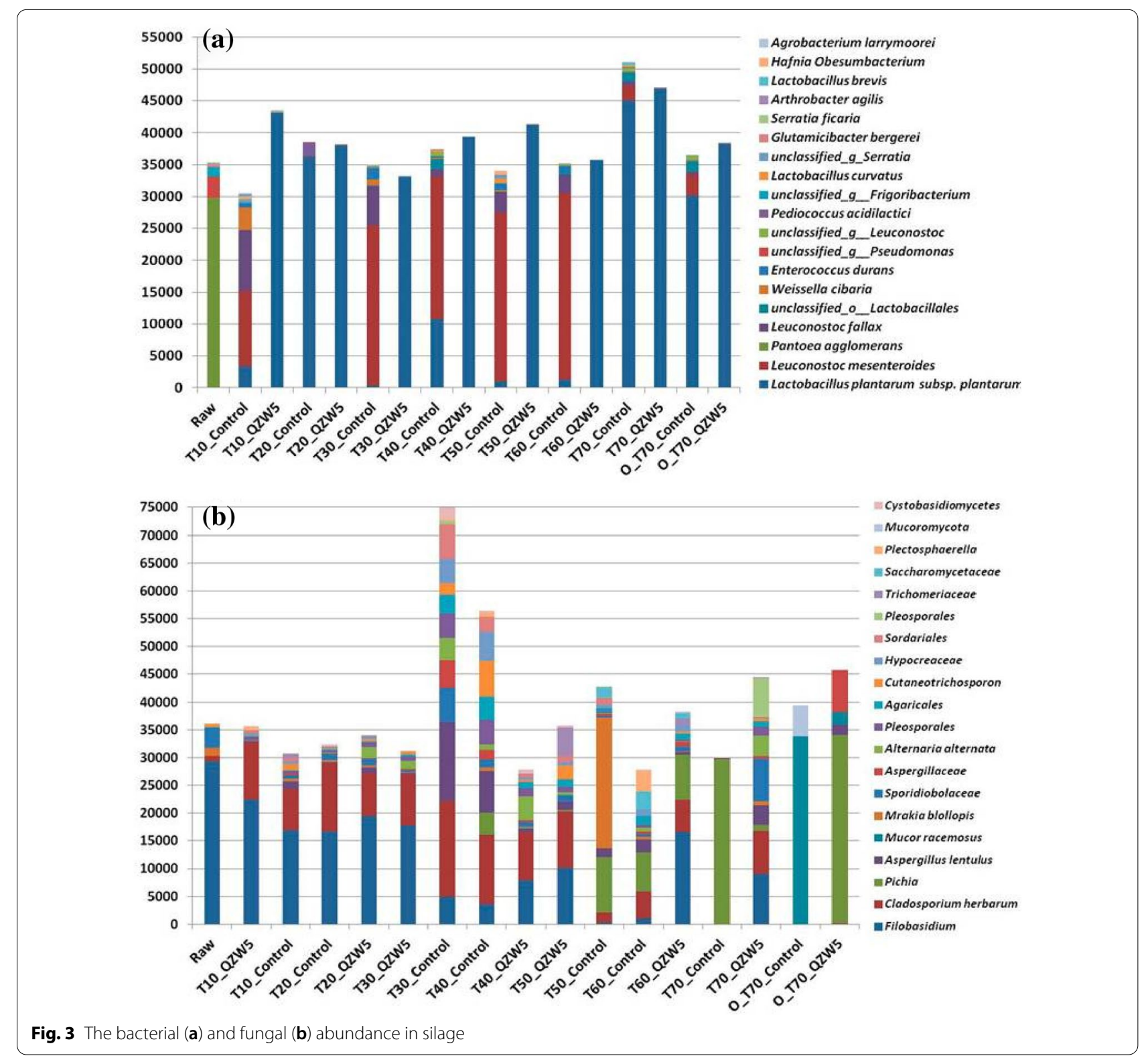



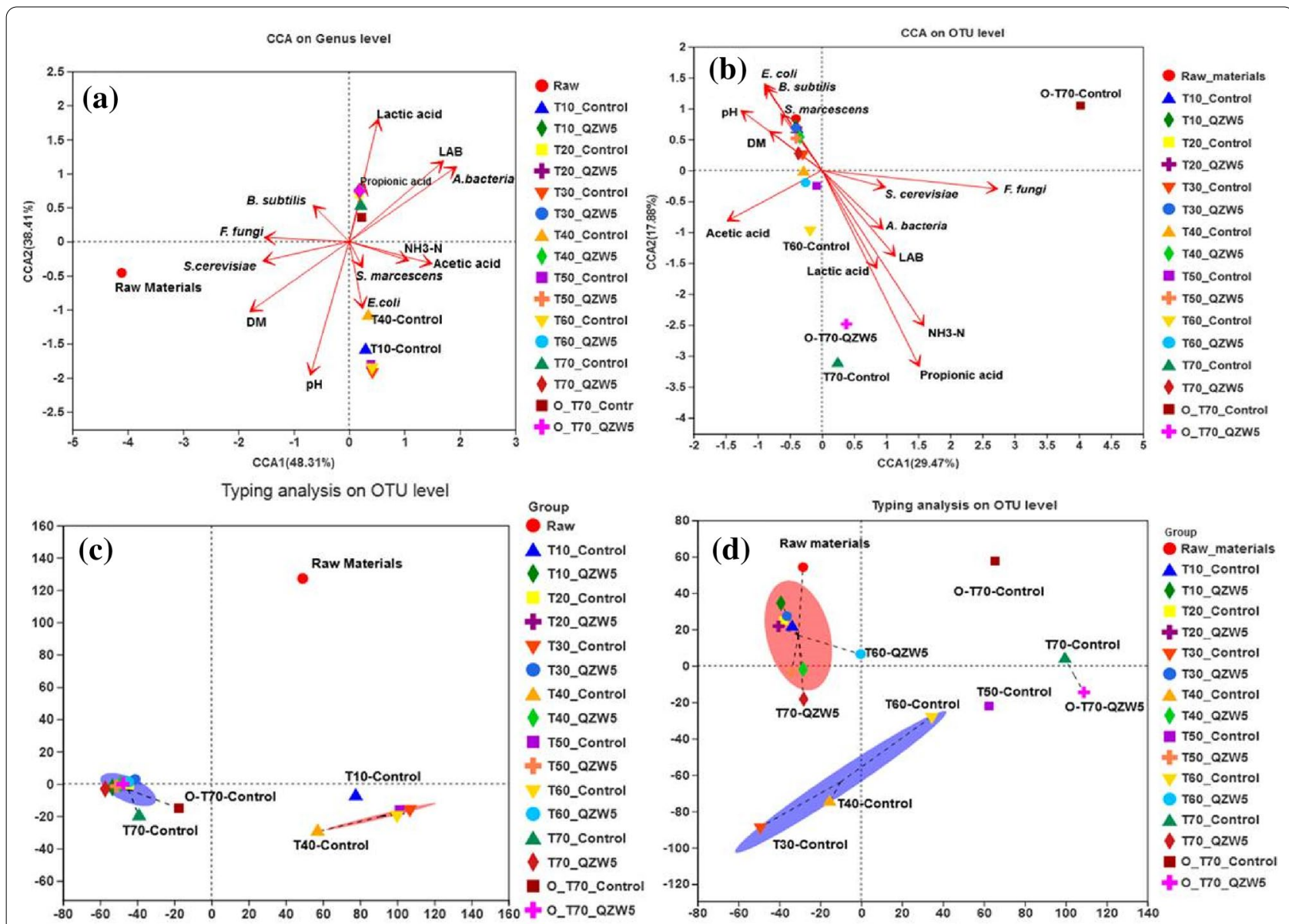

Fig. 4 CCA and typing analysis of the silage. a The CCA analysis of bacteria was performed at genus level. b The CCA of fungi on OTU level. c The typing analysis of bacteria on OTU level. $\mathbf{d}$ The typing analysis of fungi on OTU level

fermentation bacteria proliferation and altered the bacterial composition in silage. However, the predominant bacteria in the group without QZW5 was inconsistent and changed. During the subsequent freeze-thaw stage from days 30 to 60 , the dominant bacterial community in the QZW5 group was L. plantarum subsp. plantarum, while that in the control group was L. mesenteroides and Leuconostoc fallax. In the entire ensiling process, the bacterial flora in QZW5 group was stability, whereas that in the control group was unstable. With oxygen exposure and temperature increase from days 60 to days 70 , the abundance of $L$. plantarum subsp. plantarum increased in the control group both under aerobic and anaerobic conditions. Thus, it can be presumed that, combined with $\mathrm{pH}$ and organic acids variations (Table 3), L. plantarum subsp. plantarum produced organic acid, lowered the $\mathrm{pH}$, and inhibited the growth of other bacteria such as $L$. mesenteroides.

The relative abundances of fungi in the silage are shown in Fig. 3b. Filobasidium was the most abundant fungus in the unfermented materials and 10 days fermented silage. It has been reported that Mucoromycota, Aspergillus bubble, Rhizopus oryzae, Penicillium acanthopanax, and Penicillium decumbens are the common pathogenic fungi detected in deteriorated silage [27]. In our study, more fungal genera were detected in the control group after fermentation for 30 days at constant temperature. Compared with those in the QZW5 group, and the abundances of pathogenic fungi such as Aspergillus, Sporidiobolaceae, Hypocreaceae, Pleosporales, Alternaria, and Cystobasidiomycetes were higher in the control group. The results indicated that strain QZW5 inhibited these fungal pathogens under the condition of constant low temperature of $5{ }^{\circ} \mathrm{C}$.

Yeasts have been considered as the main initiators of aerobic deterioration of silage, with the process of aerobic deterioration closely related to the metabolism of the dominant yeast strain $[28,29]$. The yeasts that cause aerobic deterioration of silage can be divided into two categories. The first is acid-utilizing yeasts, such 

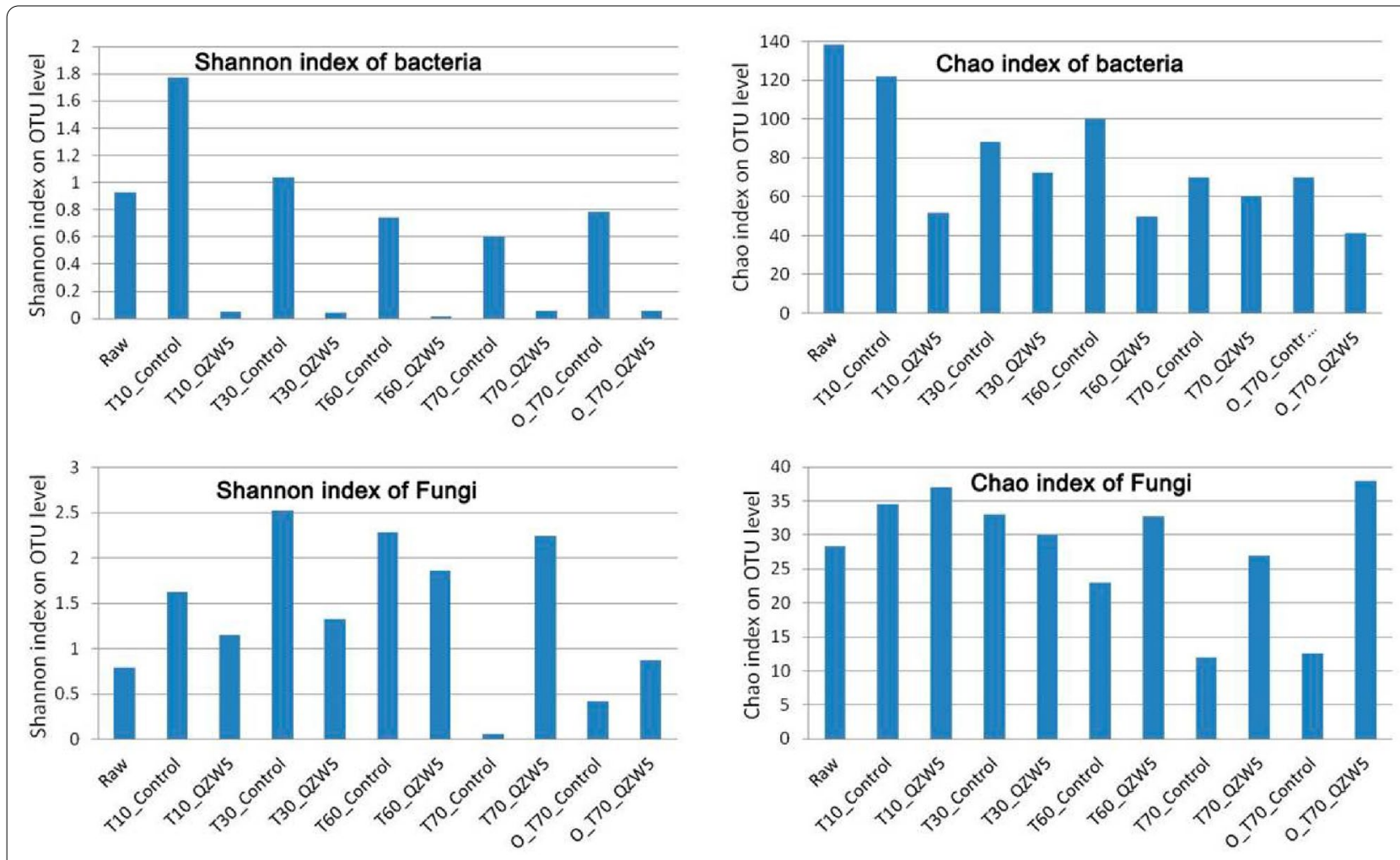

Fig. 5 Alpha diversity estimators of bacteria and fungi on OTU level

as Candida, Endomycopsis, Hansenula, and Pichia. The second is sugars-utilizing yeasts, such as Torulopsis, Pichia manshurica, Candida ethanolica, and Zygosomenevia bailii, which can tolerate acetic acid and have a greater effect on aerobic metamorphism of silage during the early fermentation stage [30]. In our study, the dominant fungus in the control group on day 70 was Pichia, which signified the initial stage of aerobic decay on days 70 . With subsequent exposure of silage to aerobic condition, Mucor racemosus and Pichia became the most abundant fungi, indicating that the quality of silage may be decreasing. Although the effects of QZW5 on the aerobic stability of silage was limited with increasing temperature, the strain QZW5 improved the stability of silage when the temperature increased,

As shown in Fig. 4a, b, the levels of lactic acid were positively correlated with the abundance of LAB. However, the $\mathrm{pH}$ value and the levels of lactic acid were negatively correlated. $\mathrm{LAB}, \mathrm{pH}$, lactic acid, aerobic bacteria, and DM significantly affected the bacterial composition in silage. The viable $F$. fungi, propionic acid, and $\mathrm{NH}_{3}-\mathrm{N}$ content significantly affected the fungal composition in silage.
Compared with control group, the $\alpha$-diversity of bacteria, such as Shannon and Chao index, showed significant lower in QZW5 group (Fig. 5). In contrast, the $\alpha$-diversity of fungi inQZW5 group decreased from day 10 to 60, but increased in day 70, when the temperature was increased to $25{ }^{\circ} \mathrm{C}$. Furthermore, the richness of fungal species in the QZW5 group was increased, when compared with that of the control group.

\section{Conclusions}

L. plantarum QZW5 has excellent tolerance to environment stress, acid production, and bacteriostasis. The $\mathrm{pH}$ of the silage of QZW5 group decreased to 4.26 on day 30 and remained at a relatively low level during the subsequent variable low-temperature stage, which can improve storage. Furthermore, lactic acid was detected in the silage of the QZW5 group, but not in the control group. Inoculation with strain QZW5 enhanced the dominant fermentation flora, reduced the abundance of fungi such as Aspergillus, Sporidiobolaceae, and Hypocreaceae at a low temperature of $5{ }^{\circ} \mathrm{C}$; decreased the abundance of fungal pathogens such as Pichia, Mucoromycota and Aspergillus under variable temperature conditions, and inhibited M. racemosus and Pichia under anaerobic 
condition at $25{ }^{\circ} \mathrm{C}$. Besides, strain QZW5 also reduced the bacterial richness (Chao index) and diversity (Shannon index) in the silage $(P<0.05)$. Most importantly, strain QZW5 improved the stability of silage subjected to multigelation, and could have potential application as a starter culture under recurrent freezing and thawing conditions. In future studies, the aerobic stability of silage under multigelation must be further investigated.

\section{Supplementary Information}

The online version contains supplementary material available at https://doi. org/10.1186/s40538-021-00251-6.

Additional file 1: Figure S1 The maximum and minimum temperatures of Hainan Zhou. Data from The weather network (http://www.weather com.cn/ )

\section{Acknowledgements}

This work was supported by the Scientific and Technological Cooperation Program for Assistance to Qinghai (Grant No. 2021-QY-204).

\section{Authors' contributions}

Conceptualization, ZT; methodology, ZT and MZ; software, MZ; validation, ZT, $\mathrm{MZ}$ and $\mathrm{YL}$; formal analysis, ZT and $\mathrm{BZ}$; investigation, $\mathrm{MZ}$; resources, $\mathrm{ZT}$; data curation, ZT and $M Z$; writing —original draft preparation, $M Z$; writing-review and editing, ZT and MZ; supervision, BZ, HP; project administration, MZ, JC and $Y L$; funding acquisition, $Z$ T; GW and LW. All authors read and approved the final manuscript.

\section{Funding}

This study was supported by the Scientific and Technological Cooperation Program for Assistance to Qinghai (Grant No. 2021-QY-204) and Scientific and Technological Cooperation Program for Assistance to Qinghai (Grant No. 2021-QY-204).

\section{Availability of data and materials}

The datasets used or analyzed during the current study are available from the corresponding author on reasonable request.

\section{Declarations}

Ethics approval and consent to participate

Not applicable.

\section{Consent for publication}

All authors listed have read the complete manuscript and have approved submission of the paper.

\section{Competing interests}

No conflicts of interest declared by the authors.

\author{
Author details \\ ${ }^{1}$ Henan Key Laboratory of Ion-Beam Bioengineering, College of Agricultural \\ Sciences, Zhengzhou University, Zhengzhou, China. ${ }^{2}$ Academy of Animal \\ Science and Veterinary Medicine, Qinghai University, Xining, China. ${ }^{3}$ Depart- \\ ment of Chemical and Environmental Engineering, Jiaozuo University, Jiaozuo, \\ China.
}

Received: 4 June 2021 Accepted: 21 Auqust 2021

Published online: 26 October 2021

\section{References}

1. Zhang M. The screening and application of psychrophilic lactic acid bacteria and the optimizing of the low-temperature-ensiling system, Henan Key Laboratory of Ion-Beam Bioengineering. Zhengzhou University; 2018.

2. Zhang M. Improving the fermentation quality of wheat straw silage stored at low temperature by psychrotrophic lactic acid bacteria. Animal Sci J Nihon Chikusan Gakkaiho. 2017;88(2):277.

3. Zhuang X, Makover J, Klauda J. Temperature dependence of bilayer structural properties studied with molecular dynamics simulations. Biophysics J. 2014;106:80a.

4. Zhang Q, et al. Effects of initial pH on heat resistance of Lactobacillus plantarum LIP-1. LWT. 2021;138:110748.

5. Yang $Y$, et al. The microbial gene diversity along an elevation gradient of the Tibetan grassland. ISME J. 2014;8(2):430.

6. Qiu J. China: the third pole. Nature. 2008:454:393-6.

7. Qiu J. A sky-high view of the third pole: an interview with Tandong Yao and Weiming Fan. Natl Sci Rev. 2015:2(4):489-92.

8. Silva VP, et al. Selection of lactic acid bacteria from alfalfa silage and its effects as inoculant on silage fermentation. Agriculture. 2020;10(11):518.

9. Tian J, et al. Interaction effect of silo density and additives on the fermentation quality, microbial counts, chemical composition and in vitro degradability of rice straw silage. Bioresour Technol. 2020;297:122412.

10. Jiang YT, Yan PF, Liang JP. Biological changes of Enterococcus faecalis in the viable but nonculturable state. Genet Mol Res. 2015:14:14790-801.

11. Pang $\mathrm{H}$, et al. Reclassification of Lactobacillus kimchii and Lactobacillus bobalius as later subjective synonyms of Lactobacillus paralimentarius. Int J Syst Evol Microbiol. 2012;62:2383-7.

12. Ni K, et al. Characterization, identification and application of lactic acid bacteria isolated from forage paddy rice silage. PLOS ONE. 2015;10(3):e0121967.

13. Zhang $M$, et al. Improving the fermentation quality of wheat straw silage stored at low temperature by psychrotrophic lactic acid bacteria. Anim Sci J. 2017;88(2):277-85.

14. Zhang $M$, et al. Ensilage of oats and wheatgrass under natural alpine climatic conditions by indigenous lactic acid bacteria species isolated from high-cold areas. PLOS ONE. 2018;13(2):e0192368.

15. Ali G, et al. Characteristics of lactic acid bacteria isolates and their effects on the fermentation quality of acacia (Sophora japonica L.) leaf silage at low temperatures. Grassland Sci. 2017:63(3):141-9.

16. Gao GF, et al. Spartina alterniflora invasion alters soil bacterial communities and enhances soil $\mathrm{N} 2 \mathrm{O}$ emissions by stimulating soil denitrification in mangrove wetland. Sci Total Environ. 2018;653:231.

17. Yang $L$, et al. Dynamics of microbial community and fermentation quality during ensiling of sterile and nonsterile alfalfa with or without Lactobacillus plantarum inoculant. Bioresour Technol. 2019;275:280-7.

18. Tharangani RMH, et al. Corn silage quality index: an index combining milk yield, silage nutritional and fermentation parameters. Animal Feed Sci Technol. 2021:273:114817

19. Ogunade IM, et al. Bacterial diversity and composition of alfalfa silage as analyzed by Illumina MiSeq sequencing: effects of Escherichia coli 0157:H7 and silage additives. J Dairy Sci. 2017;101(3):2048-59.

20. Ogunade IM et al. Control of Escherichia coli O157:H7 in contaminated alfalfa silage: effects of silage additives. J Dairy Sci. 2016;99(6):4427-36.

21. Li J-F, et al. Effect of adding acetic acid on fermentation quality and aerobic stability of mixed oat and alfalfa silage in Tibet. Acta Prataculturae Sinica. 2014;271-8.

22. Danner $\mathrm{H}$, et al. Acetic acid increases stability of silage under aerobic conditions. Appl Environ Microbiol. 2003;69(1):562-562.

23. Oliveira AS, et al. Meta-analysis of effects of inoculation with homofermentative and facultative heterofermentative lactic acid bacteria on silage fermentation, aerobic stability, and the performance of dairy cows. J Dairy Sci. 2017;100(6):4587-603.

24. Bastidas-Oyanedel JR, et al. Dark fermentation biorefinery in the present and future (bio)chemical industry. Rev Environ Sci Bio/Technol. 2015;14(3):473-98. 
25. Li J, et al. Effect of nano-magnetite on the propionic acid degradation in anaerobic digestion system with acclimated sludge. Bioresour Technol. 2021;334:125143.

26. Anésio $A H C$, et al. Effects of ensiling density on chemical and microbiological characteristics of sorghum silage. J Animal Feed Sci. 2017;26(1):65-9.

27. Ciofu O. Chromosomal beta-lactamase is packaged into membrane vesicles and secreted from Pseudomonas aeruginosa. J Antimicrob Chemother. 2000;45(1):9-13.

28. Assar Ali S. Ensiling of whole-plant hybrid pennisetum with natamycin and Lactobacillus plantarum impacts on fermentation characteristics and meta-genomic microbial community at low temperature. J Sci Food Agric. 2020;8(100):3378-85.
29. Da SRB, et al. Effects of a chemical additive on the fermentation, microbial communities, and aerobic stability of corn silage with or without air stress during storage. J Animal Ence. 2020;8:8.

30. Wang $\mathrm{H}$, et al. Characterization of culturable yeast species associating with whole crop corn and total mixed ration silage. Asian Australas J Anim Sci. 2018;2(31):198-207.

\section{Publisher's Note}

Springer Nature remains neutral with regard to jurisdictional claims in published maps and institutional affiliations.

\section{Submit your manuscript to a SpringerOpen ${ }^{\circ}$ journal and benefit from:}

- Convenient online submission

- Rigorous peer review

- Open access: articles freely available online

- High visibility within the field

- Retaining the copyright to your article

Submit your next manuscript at $\boldsymbol{\nabla}$ springeropen.com 